\title{
Formulating the in-plane frictional resistances and collapse mechanisms for multi-storey masonry block walls
}

\author{
L.U. Argiento, A. Maione, C. Casapulla \\ University of Napoli Federico II, Department of Structures for Engineering and Architecture, Italy \\ lucaumberto.argiento@unina.it \\ arch.maione@gmail.com \\ casacla@unina.it
}

\begin{abstract}
In this paper a macro-block model accounting for frictional resistances is presented to assess the lateral strength of a multi-storey masonry block wall. The kinematic approach of limit analysis is used to define the load factor causing the onset of rocking-sliding mechanism under in-plane horizontal loading. A dry frictional contact condition is assumed at the rigid block interfaces, according to the Coulomb's law with non-associated flow rule. The key aspect of the proposed approach is the introduction of a criterion to evaluate the contribution of the actual frictional resistances depending on the inclination angle of the crack line. An accurate assessment of the frictional resistances is also obtained by distinguishing two different contributions (the wall own weight and additional vertical loads) and their application points. Hence, a sensitivity analysis is performed with respect to the overloading condition, the friction coefficient, and geometrical parameters such as the shape ratios of the wall and of the unit block and the number of rows. The analytical results of the proposed model are also validated against results from other existing macro and micro-block modelling approaches in terms of load factor. The comparison confirms the reliability of the proposed model that allows, with similar results, great simplification of the computational effort with respect to micro-block models.
\end{abstract}

KEYWORDS. Limit analysis; 3D macro-block model; Multi-storey masonry building; Rocking-sliding failure; Frictional resistances.

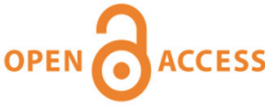

Citation: Argiento, L.U, Maione, A., Casapulla, C., Formulating the in-plane frictional resistances and collapse mechanisms for multi-storey masonry block walls, Frattura ed Integrità Strutturale, 46 (2018) 226-239.

Received: 29.07 .2018 Accepted: 26.08 .2018 Published: 01.10.2018

Copyright: (C) 2018 This is an open access article under the terms of the CC-BY 4.0, which permits unrestricted use, distribution, and reproduction in any medium, provided the original author and source are credited.

\section{INTRODUCTION}

$\mathrm{T}$ he seismic damages suffered by unreinforced masonry block structures are often due to partial collapses with loss of equilibrium of walls or portions of them, while material failure rarely occurs. Experimental tests carried out since '80s of the last century on brickwork samples [1-3] have proved that the mechanical performance of such a 
material is characterized by a very high anisotropy, so that the direction of bedding surfaces and element shapes plays a central role in defining the type of failure. Such experiments, further developed by other authors as Ceradini [4], have stressed that in-plane failure in most of cases occurs by applying horizontal loads exceeding the friction coefficient, such to produce cracks along the joints without crashing of elements, while failure due to the overcoming of material strength $(\sigma, \tau)$ occurs for much higher applied loads.

Actually, the absence of tensile strength and the presence of weak mortar joints generally cause the development of cracks which transforms a masonry block structure into a system of rigid blocks which can exhibit sliding or rocking mechanisms as well as a combination of them. The geometry of these mechanisms can be quite easily identified in post seismic scenarios, since the crack pattern has been revealed, but when the attention is focused on the prevision of the expected behaviour of the structures the most likely collapse mechanisms have to be identified.

To this aim, several combinations of material and structural models are possible, e.g. FEM macro and micro-modelling [5], discrete element methods [6, 7], computational limit analysis procedure for rigid block assemblages [8, 9] or homogenization models [10]. A drawback for the use of these sophisticated models in the practical assessment of structures is the large amount of time needed for the structural model elaboration, for performing the non-linear analyses themselves and for reaching proper understanding of the results significance.

Conversely, the macro-block modelling in the framework of limit analysis with kinematic approach represents a more powerful tool because it allows an easy computation of the collapse load factor and the failure mode by means of minimization routines. The pioneering works of Kooharian [11] and Heyman [12], based on infinite frictional resistances, have largely been used to apply the plasticity theory to masonry block structures. According to this standard limit analysis, the application of the static theorem provides a lower-bound or safe solution of the collapse load factor, based on equilibrium equations, while the application of the kinematic theorem leads to an upper bound multiplier. The solution that satisfies the hypotheses of both theorems, equilibrium, compatibility and material conditions is the correct solution and provides the collapse load multiplier for the specific problem.

However, the uniqueness of the solution is no longer guaranteed when non associated flow rules are involved, such as in case of Coulomb's friction at block interfaces [13,14]. This means that a range of statically and kinematically admissible solutions can be identified and a safe solution can be represented by the minimum load multiplier computed by means of the kinematic approach [8]. Crucial aspects are also the definition of the yield domains of dry or weak mortar joints able to dissipate seismic energy [15-17] or, as an alternative, the composition of units to enforce the capability of dissipating energy during motions of walls [18].

The macro-block modelling approach has been lately developed to investigate the local in-plane and out-of-plane failure modes in masonry buildings [19-22]. According to it, each block represents a portion of masonry which remains undamaged and is separated from others by a number of localized cracking where the frictional resistances can take place. When attention is paid to structural elements having a specific role in the overall building behaviour, special macroelements can simulate them to reduce computational and modelling efforts, e.g. architectonic elements in which the seismic behaviour is almost independent from the rest of the structure of masonry churches [23, 24] or masonry vaults typified by sets of equivalent trusses [25]. This modelling strategy could also be useful to further develop recent innovative research in the field of rocking rigid block dynamics [26, 27].

When applied to in-plane failure modes of masonry block structures with dry or weak mortar joints, generally characterized by a combined rocking-sliding mechanism, crucial to this approach is the assessment of the frictional resistances along the crack. In fact, it is not easy to identify the number of active sliding interfaces along the crack and the actual frictional resistance associated to the crack line could also be far from its maximum value. This issue has already been addressed with reference to a single-storey masonry block wall [22] and in this paper it is extended to a multi-storey wall. A proper evaluation of the in-plane frictional resistances, on the other hand, represents a relevant contribution also to the analysis of the out-of plane mechanisms; in fact these resistances can play an important role when the mechanism involves the façade wall with portions of side walls of a masonry building (complex rocking).

Thus, in the following sections, considering the masonry block wall as a single leaf wall arranged in a running bond pattern, the in-plane frictional resistances are firstly evaluated, also accounting for additional loads due to horizontal structures and live loads. The originality of this part can be recognized in the criterion to evaluate the contribution of the actual frictional resistances depending on the inclination angle of the crack line and the number of storeys, in addition to other geometrical and mechanical parameters. Then, the "exact" ultimate load factor in case of rocking-sliding failure mode is computed by means of minimization routines, by taking into account variable positions of the conventional crack line. Lastly, the validity and accuracy of the novel solution procedure are investigated through a sensitivity analysis and the comparison with results and benchmark examples existing in the literature. 


\section{IN-PLANE FRICTIONAL RESISTANCES OF A MULTI-STOREY MASONRY WALL}

7 he analysis of the rocking-sliding failure mode of a multi-storey wall is an extension of that referred to a singlestorey wall, based on a simple geometric and mechanical model.

It is assumed, in fact, that the single-storey wall is made up of a single leaf of regular squared and rigid blocks, with geometric dimensions $l \times b \times b$ (Fig. 1a), placed with their longer side parallel to the wall length and overlapped with constant staggering length $v$, equal to half the block length (Fig. 1b). The blocks interact through their bed joints according to the Coulomb's model of dry frictional contact [13]; no tensile strength, instead, is assumed against vertical loads which tend to detach overlapping layers.
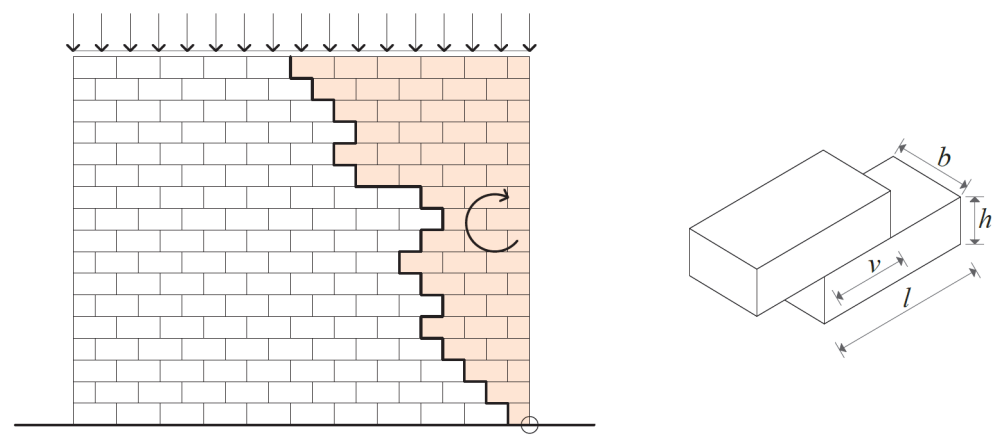

Figure 1: a) Masonry wall with dry assembled unit blocks; b) dimensions of the unit block.

In this section the criterion developed and validated in previous works [21, 22] is applied to a multi-storey masonry wall to define the in-plane frictional resistances involved in the combined rocking-sliding mechanism. The adoption of macromodelling approach implies that the damage pattern is schematized by a single crack where the relative motions between the macro-blocks and frictional resistances develop.

The difficulty of identifying the number of active sliding interfaces along the crack in a combined mechanism has been overcome by introducing a reduction criterion of the maximum value of the resultant frictional resistance based on the inclination of the crack. In fact, as highlighted in [22], this inclination tends to become vertical when pure sliding occurs and the resultant frictional resistance assume the maximum value; on the other hand, instead, the inclination tends to the staggering ratio when pure rocking occurs with no frictional resistances. Among these two limit cases, a reliable evaluation of the resultant frictional resistance related to a combined mechanism can be found, as described in the following.

According to the proposed approach, the maximum value of the resultant frictional resistance is firstly calculated assuming a pure sliding mechanism.

Hence, with reference to the generic multi-storey wall in Fig. 2 with dimensions $L \times H$ and $N$ storeys of heights $H_{i}$ (storey 1 is at the top wall), the following parameter is defined:

$$
\tan \alpha_{p i}=\frac{L}{\sum_{j=i}^{N} H_{j}}
$$

which represents the shape ratio of the portion of the wall between the hinge position and the top storey $i$. It is worth noting that it is also $\tan \alpha_{p 1}=\tan \alpha_{p}=L / H$. The parameter $H_{c i}$, instead, represents the height of the portion of the wall related to the storey $i$, which is crossed by the crack line. In fact, depending on the position of the crack line, inclined of the angle $\alpha_{c}, H_{c i}$ can assume the following expressions:
a) $\quad \alpha_{c} \leq \alpha_{p i}$
$H_{c i}=H_{i}=n_{i} h$
b) $\quad \alpha_{p i} \leq \alpha_{c} \leq \alpha_{p i+1}$
$H_{c i}=\frac{L}{\tan a_{c}}-\sum_{j=i+1}^{N} n_{j} b$
c) $\quad \alpha_{c} \geq \alpha_{p i+1}$
$H_{c i}=0$ 
where $n_{i}$ is the number of rows related to the storey $i\left(n_{i}=H_{i} / h\right.$, being $b$ the height of the unit block). The parameter $H_{c i}$ can also be expressed as a function of the number $n_{c i}$ of rows crossed by the crack line at the storey $i$, i.e:

$$
H_{c i}=n_{c i} b
$$

It is worth noting that the cases in Eq. (2) cover all the possible configurations of the analyzed mechanism, and the geometric parameter $H_{c}$, or the related $n_{c}$, easily allows extending the relations defining the model of the single-storey wall to the multi-storey wall, as will be better shown later. The use of the relations defined for the single-storey wall in a previous work [22], instead, would have required a large number of conditions on the same relations, sharply increasing with the number of the stories.

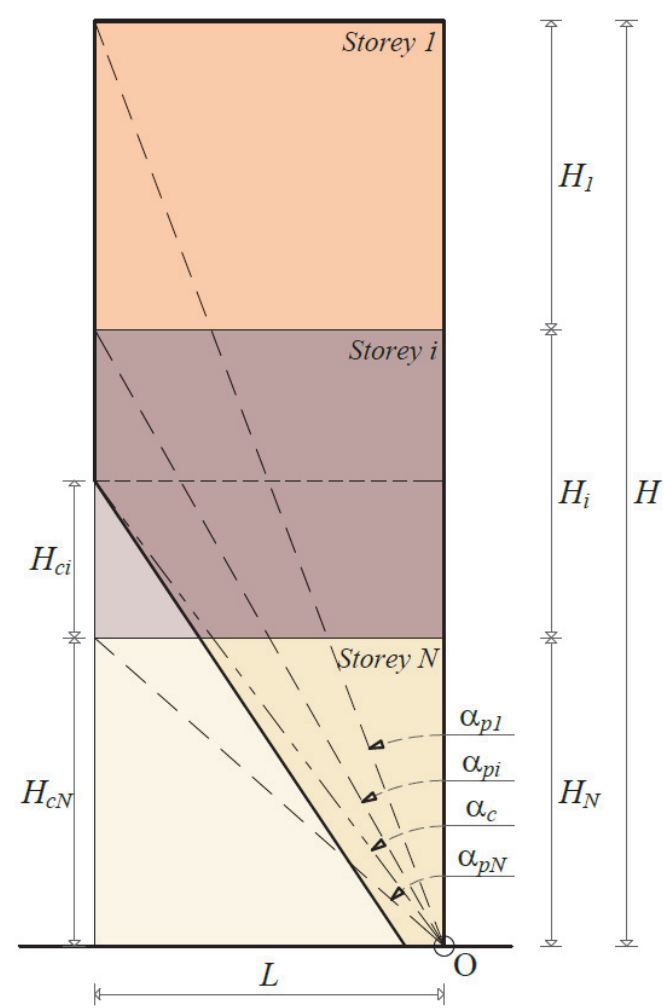

Figure 2: Macro-block model of a multi-storey wall with identification of the masonry panels involved in a rocking-sliding mechanism.

The resultant of the frictional resistances is obtained as sum of two components in order to take into account the effect of their different points of application on the stabilizing moment. In particular, with reference to the storey $i$ (Fig. 3), the component $F_{g i}$ is due to the self-weight of the moving portion of the wall crossed by the crack and corresponds to a linear distribution along the height $H_{c i}$; the component $F_{q i}$, instead, is due to the overloading and to the self-weight of the portion of the wall with height $H-L / \tan \alpha_{c}$ and corresponds to a uniform distribution along the height $H_{c i}$. These components are here expressed by adopting the discrete formulation based on the number $n_{i i}$ of rows crossed by the crack line at the storey $i$.

Thus, the frictional force $S_{k}$ at contact interface $k$ is given by the weight $W_{b i}$ of the upper column of half-blocks, limited to the height of the crack line $H_{c i}$, multiplied by the friction coefficient $f$ :

$$
S_{k}=k f W_{b i} \quad\left(k=0,1, \ldots, n_{c i}\right)
$$

where $W_{b i}=\gamma v b b_{i}, b_{i}$ is the thickness of the unit block related to the level $i$ (it could also be different from those at different levels) and $\gamma$ is the specific weight of masonry.

The two components $F_{g i}$ and $F_{q i}$ respectively are: 


$$
\begin{aligned}
& F_{g i}=\sum_{k=1}^{n_{i j}} S_{k}=v b b_{i} \frac{n_{c i}\left(n_{c i}+1\right)}{2} \gamma f \\
& F_{q i}=\left(\left(n_{i}-n_{c i}\right) b_{i} b \gamma+\sum_{j=1}^{i} q_{j}+\sum_{j=1}^{i-1} n_{j} b_{j} b \gamma\right) n_{c i} v f
\end{aligned}
$$

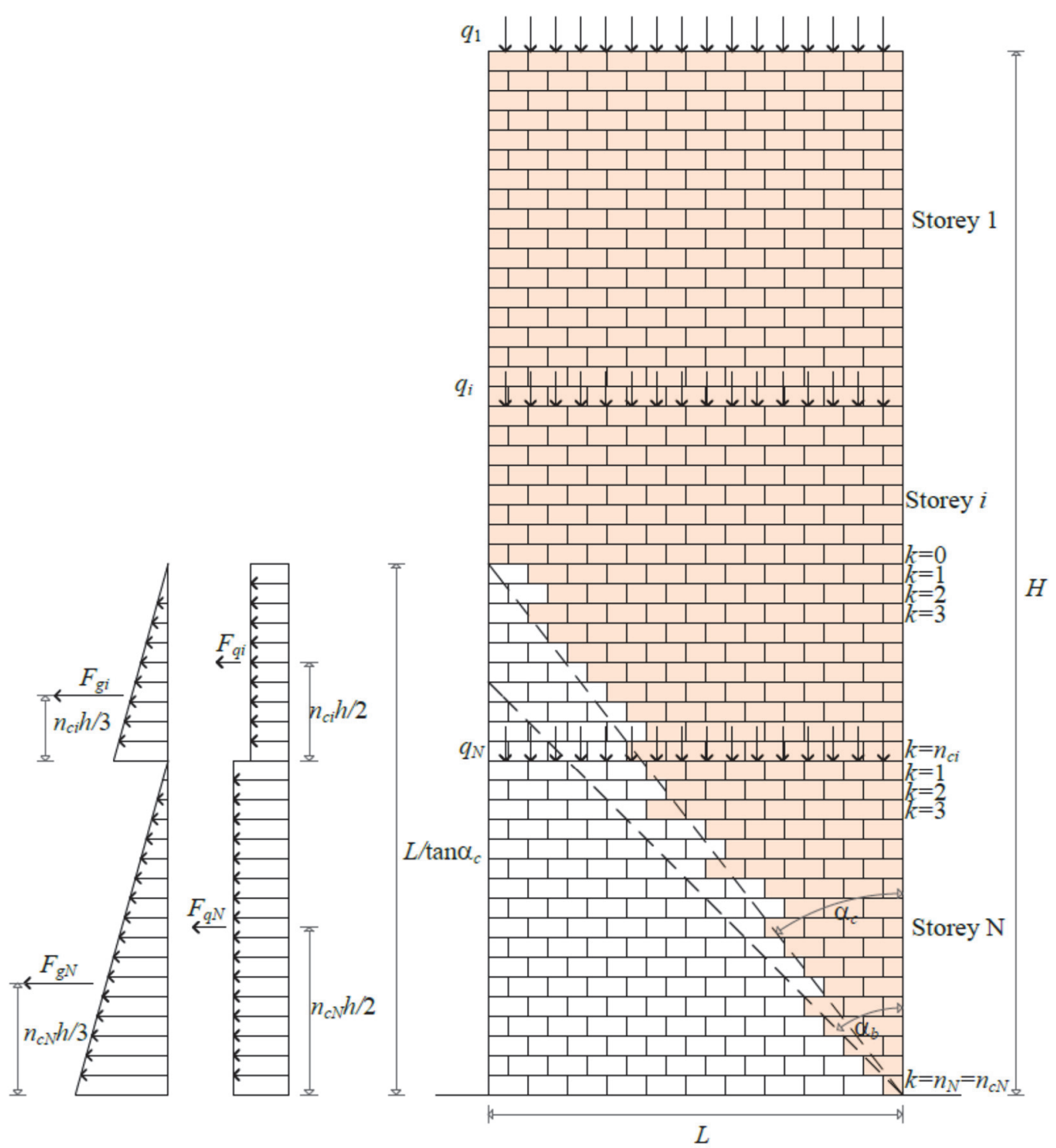

Figure 3: Frictional resistances related to a multi-storey wall.

It is easy to verify [22] that $F_{g i}$ is applied at $2 / 3$ from the top of the height $H_{c i}$ since $S_{k}$ linearly increases from the top to the bottom, while $F_{q i}$ is applied at the half of the same height $H_{i i}$.

Hence, if a full activation of the frictional resistances occurs along the all the bed joints crossed by the crack line (pure sliding mechanism), their resultant assumes a maximum value given by:

$$
F=\sum_{i=1}^{N} F_{g i}+\sum_{i=1}^{N} F_{q i}
$$


If a combined rocking-sliding mechanism takes place, instead, this force is expected to be lower than the value provided by Eq. (7); in this case, in fact, the rocking motion causes the uplift of a number of blocks and the reduction of the contact interfaces. In order to overcome the difficulty of identifying the real contact conditions, a criterion to assess the actual frictional resistances has been proposed and validated by Casapulla et al. [21]. This criterion, as previously introduced, is based on the inclination of the crack line and provides a reliable estimation of the frictional resistances $F_{W}$ related to a combined mechanism. It is assumed in fact:

$$
F_{W}=F\left(1-\frac{\alpha_{c}}{\alpha_{b}}\right)
$$

where $\alpha_{b}=\tan ^{-1}(v / h)$ depends on the dimensions of the unit block (Fig. 1b). Hence, when the crack line is vertical $\left(\alpha_{c}=0\right)$, all the contact interfaces along the crack are involved in sliding, so that the frictional resistances can be considered as activated on all courses crossed by the crack and their resultant attains its maximum value; in fact, it is $F_{W}=F$, with $F$ given by Eq. (7). When $\alpha_{c}=\alpha_{b}$, instead, a pure rocking mechanism takes place with loss of contact over all the involved joints; as a consequence, the resultant frictional resistance can be considered null $\left(F_{W}=0\right)$. When $0<\alpha_{c}<\alpha_{b}$, a combined rocking-sliding mechanism is expected with a resultant frictional resistance lower than its maximum value $\left(0<F_{W}<F\right)$. It is worth noting that the case $\alpha_{c}>\alpha_{b}$ it is excluded because unrealistic for dry joint masonry walls.

Finally, in order to better understand the proposed model, in Tab. 1 the parameter $H_{c i}$ and the components $F_{g i}$ and $F_{q i}$ defined in a compact form by Eqs. (3), (5) and (6), respectively, are explicated with reference to a number of storey $i=3$ and $\alpha_{p 2}<\alpha_{c}<\alpha_{\mathrm{p} 3}$ (Figs. 2 and 3).

It is worth noting that $H_{c 1}, F_{g 1}$ and $F_{q 1}$ are all null because the crack line does not cross the wall at the storey 1 . Moreover, being $\alpha_{p 2}<\alpha_{c}<\alpha_{p 3}$, Eqs. (2b) and (2c) are used to define $H_{c 2}$ and $H_{c 3}$, respectively.

\begin{tabular}{cccc}
\hline Storey & $H_{c i}$ Eq. (2) & $F_{g i}$ Eq. (5) & $F_{q i}$ Eq. (6) \\
1 & - & - & - \\
2 & $\frac{L}{\tan \alpha_{c}}-\left(n_{2}+n_{3}\right) b$ & $v b b_{2} \frac{n_{c 2}\left(n_{c 2}+1\right)}{2} \gamma f$ & $\left(\left(n_{2}-n_{c 2}\right) b_{2} h \gamma+q_{1}+q_{2}+n_{1} b_{1} b \gamma\right) n_{c 2} v f$ \\
3 & $n_{3} h$ & $v b b_{3} \frac{n_{3}\left(n_{3}+1\right)}{2} \gamma f$ & $\left(q_{1}+q_{2}+q_{3}+\left(n_{1} b_{1}+n_{2} b_{2}\right) b \gamma\right) n_{c 3} v f$ \\
\hline
\end{tabular}

Table 1: Expressions of the variables $H_{i,}, F_{g i}$ and $F_{q i}$ for a three-storey masonry wall, when $\alpha_{p 2}<\alpha_{c}<\alpha_{p 3}$.

\section{IN-PLANE FAILURE MODE OF MASONRY MULTI-STOREY WALLS AND COLLAPSE LOAD FACTORS}

he analysis of the rocking-sliding failure mode of a multi-storey wall is an extension of that referred to a singlestorey wall, based on a simple geometric and mechanical model.

The kinematic approach of the limit analysis is adopted to identify the geometry of the rocking-sliding mechanism (i.e. the inclination $\tan \alpha_{c}$ of the crack line) which minimizes the load factor causing the onset of the mechanism.

With reference to a generic multi-storey wall, Fig. 4 represents the macro-block identified by the crack line with inclination $\alpha_{c} \leq \alpha_{b}$ and the actions (internal and external) involved in the mechanism at each level $i$. These actions are applied to the centre of gravity of each of the parts in which the moving macro-block has been geometrically divided; in particular, this partition takes into account the overlapping length $v$ of the unit blocks as the width of the part $A$, marked in grey in Fig. 4 and, as a consequence, the angle $\alpha_{c}^{*}$ is used instead of $\alpha_{c}$ (Figs. 3 and 4). The relation between the parameters tan $\alpha_{c}$ and $\tan \alpha_{c}^{*}$ is:

$$
\tan \alpha_{c}^{*}=\tan \alpha_{c}-\frac{v}{n b}
$$


The external loadings together with internal frictional resistances and the coordinates of the relative application points are reported in Tab. 2.

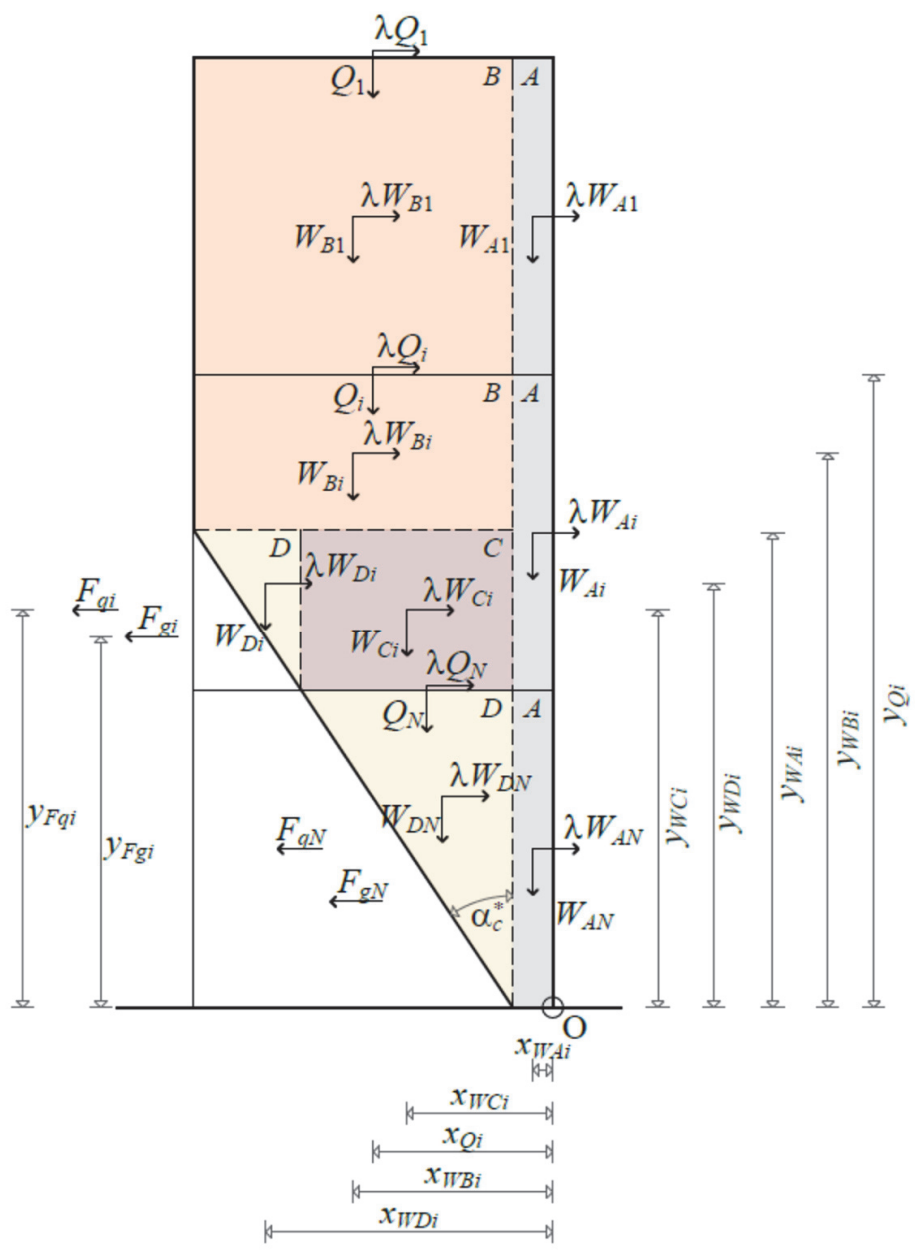

Figure 4: Representation of the external and internal actions involved in the rocking-sliding mechanism of a multi-storey masonry wall.

Thereafter, by applying the Principle of Virtual Work to the system of virtual displacements derived by the rotation around the hinge $\mathrm{O}$ of the macro-block identified by the crack line, the following expression of the load factor is obtained (Fig. 4):

$$
\lambda_{r}=\frac{\sum_{i=1}^{N} W_{A i} x_{W A i}+\sum_{i=1}^{N} W_{B i} x_{W B i}+\sum_{i=1}^{N} W_{C i} x_{W C i}+\sum_{i=1}^{N} W_{D i} x_{W D i}+\sum_{i=1}^{N} Q_{i} x_{Q i}+\sum_{i=1}^{N} F_{g i} y_{F g i}+\sum_{i=1}^{N} F_{q i} y_{F q i}}{\sum_{i=1}^{N} W_{A i} y_{W A i}+\sum_{i=1}^{N} W_{B i} y_{W B i}+\sum_{i=1}^{N} W_{C i} y_{W C i}+\sum_{i=1}^{N} W_{D i} y_{W D i}+\sum_{i=1}^{N} Q_{i} y_{Q i}}
$$

This load factor, accounting for Eq. (9) is a function of the parameter $\tan \alpha_{c}$ which defines the geometry of the mechanism; thus it can be minimized with respect to this parameter, with the following constraint:

$$
\frac{v}{n h} \leq \tan \alpha_{c} \leq \tan \alpha_{b}
$$

It is worth noting that the minimization process has to take into account the circumstance that the position of the hinge can be located at the edge of each level of the multi-storey wall causing the onset of different mechanisms; thus the load factors related to all possible positions the of the hinge have to be compared in order to choose the minimum value 


\begin{tabular}{|c|c|c|}
\hline External actions & Horizontal lever arm $\left(x_{j}\right)$ & Vertical lever arm $\left(y_{j}\right)$ \\
\hline$W_{A i}=n_{i} b_{i} b v \gamma$ & $\frac{v}{2}$ & $\left(\frac{n_{i}}{2}+\sum_{j=i+1}^{N} n_{j}\right) h$ \\
\hline$W_{B i}=\left(n_{i}-n_{i i}\right) \cdot(L-v) b b_{i} \gamma$ & $\frac{L+v}{2}$ & $\left(\frac{n_{i}+n_{c i}}{2}+\sum_{j=i+1}^{N} n_{j}\right) b$ \\
\hline$W_{C i}=\tan \alpha_{c}^{*} n_{c i} b^{2} b_{i} \cdot \gamma \sum_{j=i+1}^{N} n_{j}$ & $\left(b \frac{\tan \alpha_{c}^{*}}{2} \sum_{j=i+1}^{N} n_{j}\right)+v$ & $\left(\frac{n_{c i}}{2}+\sum_{j=i+1}^{N} n_{j}\right) b$ \\
\hline$W_{D i}=\frac{\tan \alpha_{c}^{*}\left(n_{c i} h\right)^{2}}{2} \cdot b_{i} \gamma$ & $\frac{\tan \alpha_{c}^{*} n_{c i} h}{3}+b \tan \alpha_{c}^{*} \sum_{j=i+1}^{N} n_{j}+v$ & $\left(\frac{2 n_{i i}}{3}+\sum_{j=i+1}^{N} n_{j}\right) h$ \\
\hline$\alpha_{c} \geq \alpha_{p i}$ & $\alpha_{c} \geq \alpha_{p i}$ & \\
\hline$\alpha_{c}<\alpha_{p i} \quad Q_{i}=\sum_{j=i}^{N} n_{j} b \tan \alpha_{c} q_{i}$ & $\alpha_{c}<\alpha_{p i} \quad \sum_{j=i}^{N} \frac{n_{j} b \tan \alpha_{c}}{2}$ & $\sum_{j=i}^{N} n_{j} h$ \\
\hline Internal actions & Horizontal lever $\operatorname{arm}\left(x_{j}\right)$ & Vertical lever arm $\left(y_{j}\right)$ \\
\hline$F_{g i}=v b b_{i} \frac{n_{c i}\left(n_{c i}+1\right)}{2} \gamma f$ & - & $\left(\frac{n_{c i}}{3}+\sum_{j=i+1}^{N} n_{j}\right) h$ \\
\hline$F_{q i}=\left(\left(n_{i}-n_{c i}\right) b_{i} b \gamma+\sum_{j=1}^{i} q_{j}+\sum_{j=1}^{i-1} n_{j} b_{j} b \gamma\right) n_{c i} v f$ & - & $\left(\frac{n_{i i}}{2}+\sum_{j=i+1}^{N} n_{j}\right) b$ \\
\hline
\end{tabular}

Table 2: Analytical expressions of the external and internal actions together with their lever arms.

Moreover, from the condition of translational equilibrium it is possible to define the load factor $\lambda_{s}$ related to the mechanism of pure sliding; it is:

$$
\lambda_{s}=\frac{\sum_{i=1}^{N} F_{g i}+\sum_{i=1}^{N} F_{q i}}{\sum_{i=1}^{N} W_{A i}+\sum_{i=1}^{N} W_{B i}+\sum_{i=1}^{N} W_{C i}+\sum_{i=1}^{N} W_{D i}+\sum_{i=1}^{N} Q_{i}}
$$

As already discussed in [22] it is always $\lambda_{s} \geq f$, resulting in particular $\lambda_{\mathrm{s}}=f$ when $\tan \alpha_{c}=\tan \alpha_{b}$. This implies that the pure sliding failure rarely occurs, although the following parameters can increase the vulnerability to this kind of mechanism: the slenderness of the unit blocks together with the absence of overloading, a low number of rows or a low value of the friction coefficient $[19,28]$. In these cases the entire wall is involved in the mechanism and the load factor tends to its maximum value coincident with the friction coefficient. This means that the friction coefficient represents an upper bound of the load factor and the condition $\lambda_{s}=f$ marks the transition from the mixed rocking-sliding to pure sliding mechanism.

Lastly, Tab. 3 reports the expressions of the external actions for a three-storey masonry wall, when $\alpha_{p 2}<\alpha_{c}<\alpha_{\mathrm{p} 3}$.

It can be derived from Fig. 4 that the forces $W_{C 1}$ and $W_{\mathrm{D} 1}$ at storey 1 and $W_{B 3}$ and $W_{C 3}$ at storey 3 are null. Finally, being $\alpha_{c}$ greater both than $\alpha_{p 1}$ and $\alpha_{p 2}, Q_{1}$ and $Q_{2}$ assume the maximum value depending on the length $L$ of the wall; $Q_{3}$, instead, depends on the inclination of the crack line $\alpha_{c}$, being $\alpha_{c} \leq \alpha_{p 3}$. 


\begin{tabular}{|c|c|c|c|c|c|}
\hline Storey & $W_{A i}$ & $W_{B i}$ & $W_{C i}$ & $W_{D i}$ & $Q_{i}$ \\
\hline 1 & $n_{1} b_{1} b v \gamma$ & $n_{1} \cdot(L-v) b b_{1} \gamma$ & - & - & $L q_{1}$ \\
\hline 2 & $n_{2} b_{2} h v \gamma$ & $\left(n_{2}-n_{c 2}\right) \cdot(L-v) b b_{2} \gamma$ & $\tan \alpha_{c}^{*} n_{c 2} b^{2} b_{2} \gamma n_{3}$ & $\frac{\tan \alpha_{c}^{*}\left(n_{c 2} h\right)^{2}}{2} \cdot b_{2} \gamma$ & $L q_{2}$ \\
\hline 3 & $n_{3} b_{3} h v \gamma$ & - & - & $\frac{\tan \alpha_{c}^{*}\left(n_{c 3} h\right)^{2}}{2} \cdot b_{3} \gamma$ & $n_{3} h \tan \alpha_{c} q_{3}$ \\
\hline
\end{tabular}

Table 3: Expressions of the external actions for three-storey masonry wall, when $\alpha_{p 2}<\alpha_{c}<\alpha_{p 3}$.

\section{SENSITIVITY ANALYSIS AND VALIDATION OF THE PROPOSED MODEL}

\section{Sensitivity analysis}

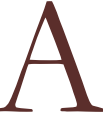
numerical analysis is here reported in order to test the sensitivity of the results obtained from the proposed model with respect to the main geometrical parameters, the overloading and the friction coefficient. The dimensionless definition of such parameters is reported in Tab. 4, together with their reference values. All the results of the numerical analysis of this and subsequent section have been obtained by using the SOLVER tool from Microsoft EXCEL to calculate the minimum of Eq. (10).

\begin{tabular}{lccc}
\hline \multicolumn{1}{c}{ Parameter } & Variable & Definition & Reference value \\
Wall shape ratio & $t$ & $H / L$ & 1 \\
Unit shape ratio & $m$ & $b / l$ & $1 / 3$ \\
Number of rows & $o$ & $H / b$ & 60 \\
Overloading ratio & $p$ & $q /(H b \gamma)$ & 0 \\
Friction coefficient & $f$ & $f$ & 0.6 \\
\hline
\end{tabular}

Table 4: Non-dimensional parameters accounted by numerical analysis.

The sensitivity analysis has been performed by varying only one parameter at a time, while keeping the others as reference values. For each parameter, three values are considered as representative of most recurrent cases.

The analyzed model is a three-storey wall, with constant inter-storey height and equal overloading condition at each level. In Tab. 5 the results in terms of load factor $\lambda$ and inclination angle $\alpha_{c}$ of the crack line are reported, highlighting the percentage variation of $\lambda$ for each set with respect to that corresponding to the reference value of the same parameter. This table also reports the $\alpha_{c} / \alpha_{b}$ ratio for each set which is useful to indicate the prevalence of a mechanism over the other; in fact, according to Eq. (8), when $\alpha_{c} / \alpha_{b} \rightarrow 1 F_{W} \rightarrow 0$ and only rocking occurs, while when $\alpha_{c} / \alpha_{b} \rightarrow 0 F_{W} \rightarrow F$ and only sliding may take place.

Overall, a relevant trend of the position of the rotational hinge emerges from the analysis. In fact, for all the accounted combinations of parameters, except for Set 4 only, the hinge is always located at the base vertex of the entire wall, defining a mechanism that always involves three levels. This also means that the load factor corresponding to such a position is always lower than those obtained if the hinge is located at the upper levels. Set 4 is the only case with the hinge at the base of the top level.

Then, looking at the results related to Set 1 and 2 in Tab. 5, it arises that increasing friction coefficient implies the increment of the load factor and also of the inclination angle of the crack line with a bigger portion of the wall involved into the mechanism.

The results related to the parameters $p$ (Sets 3 and 4) and $o$ (Sets 5 and 6), show that the variation of the overloading ratio and the number of rows are substantially irrelevant both on the load factor and the angle $\alpha_{c}$. However, as previously mentioned, the only effect observed for Set 4 , corresponding to overloading ratio $p=8$, is the shift of the position of the 
hinge from the base of the three-storey wall to the base of the top wall, even if the load multipliers corresponding to the three possible positions are very close to each other $(\lambda=0.576,0.578$ and 0.582 from the top to the bottom, respectively).

\begin{tabular}{|c|c|c|c|c|c|c|}
\hline Variable & Set & Value & $\lambda$ & $\% \lambda$ Var. & $\boldsymbol{\alpha}_{c}(\mathrm{deg})$ & $\alpha_{c} / \alpha_{i}$ \\
\hline \multirow{3}{*}{$f$} & 1 & 0.4 & 0.519 & $-10.84 \%$ & 35.51 & 0.63 \\
\hline & $\operatorname{Ref}$ & 0.6 & 0.583 & - & 40.15 & 0.71 \\
\hline & 2 & 0.8 & 0.626 & $7.48 \%$ & 43.34 & 0.77 \\
\hline \multirow{3}{*}{$p$} & $\operatorname{Ref}$ & 0 & 0.583 & - & 40.15 & 0.71 \\
\hline & 3 & 2 & 0.578 & $-0.75 \%$ & 40.11 & 0.71 \\
\hline & 4 & 8 & 0.576 & $-1.20 \%$ & 40.12 & 0.71 \\
\hline \multirow{3}{*}{0} & 5 & 36 & 0.513 & $-11.94 \%$ & 37.42 & 0.66 \\
\hline & 6 & 45 & 0.585 & $0.46 \%$ & 40.16 & 0.71 \\
\hline & $\operatorname{Ref}$ & 60 & 0.583 & - & 40.15 & 0.71 \\
\hline \multirow{3}{*}{$t$} & $\operatorname{Ref}$ & 1 & 0.583 & - & 40.15 & 0.71 \\
\hline & 7 & 2 & 0.406 & $-30.30 \%$ & 55.64 & 0.99 \\
\hline & 8 & 3 & 0.290 & $-50.15 \%$ & 56.31 & 1 \\
\hline \multirow{3}{*}{$m$} & $\operatorname{Ref}$ & $1 / 3$ & 0.583 & - & 40.15 & 0.71 \\
\hline & 9 & $1 / 2$ & 0.451 & $-22.65 \%$ & 35.12 & 0.78 \\
\hline & 10 & 1 & 0.252 & $-56.72 \%$ & 26.57 & 1 \\
\hline
\end{tabular}

Table 5: Results of sensitivity analysis.

A significant influence on the load factor, instead, is due to the wall shape ratio $t$. In fact, increasing value of $t$ implies increasing slenderness of the wall and decreasing load factors $\lambda$. In particular, assuming as a reference the load factor related to a square wall $(t=1), \lambda$ decreases by about $30 \%$ when $t=2$ (Set 7 ) and by about $50 \%$ when $t=3$ (Set 8 ). Moreover, it is worth noting that if the slenderness of the wall increases, the geometry of the mechanism changes significantly with respect to the case of square wall, because it results $\alpha_{c}>\alpha_{p}$, being in particular $\alpha_{p 2}<\alpha_{c}<\alpha_{p 3}$ and $\alpha_{c}>\alpha_{p 3}$ for Sets 7 and 8 , respectively. This circumstance can justify the drastic variation of the load factor when going from $t=1$ to $t=2$, while from $t=2$ to $t=3$ the decrement of the load factor is lower.

Finally, Sets 9 and 10 reveal that also the variation of the unit shape ratio $m$ has a relevant effect both on the load factor and the angle $\alpha_{c}$. In particular, with respect to the reference value related to $m=1 / 3$, the load factor decreases by about $23 \%$ for $m=1 / 2$ and by about $57 \%$ for $m=1$. The same trend is also reported for the inclination angle of the crack line, that decreases with increasing values of $m$; in particular when $m=1$, it is $\alpha_{c}=\alpha_{b}=\tan ^{-1}(1 / 2 m)$ pointing out that in this case the frictional resistances are null and the mechanism involves only pure rocking. On the other hand, the increment of $m$ implies the reduction of the overlapping length $v$ of the unit blocks and as a consequence the reduction of the frictional resistances.

\section{Validation of the proposed model}

In this section the results provided by the proposed approach to the analysis of the in-plane rocking-sliding mechanism of a multi-storey masonry wall are compared and validated throughout the comparison with other models available in the literature, such as the micro-block model developed by Orduña [20] and the macro-block models proposed by Buhan and De Felice [29], Orduña [20] and Speranza [28]. The micro-block model, in particular, is here assumed as a reference to evaluate the macro-block models.

The non-dimensional parameters accounted in the present analysis are those reported in Tab. 4, except for the friction coefficient which is not included in the analysis and is kept as $f=0.75$ for all the sets. The different sets of values assumed 
for these parameters are reported in Tab. 6 where the geometric ones have been chosen accounting for the condition $\alpha_{c} \leq \min \left(\alpha_{b}, \alpha_{p}\right)$.

\begin{tabular}{cccccc}
\hline Set & Wall & $t$ & $m$ & $o$ & $p$ \\
11 & Single-storey & 0.5 & $1 / 3$ & 12 & - \\
12 & Single-storey & 1.0 & $1 / 3$ & 12 & - \\
13 & Single-storey & 1.0 & $1 / 2$ & 12 & - \\
14 & Single-storey & 1.0 & 1 & 12 & - \\
15 & Five-storey & 1.0 & $1 / 3$ & 150 & - \\
16 & Five-storey & 1.0 & $1 / 3$ & 150 & 1 \\
17 & Five-storey & 1.0 & $1 / 3$ & 150 & 8 \\
\hline
\end{tabular}

Table 6: Sets of values assumed by the parameters of the comparative analysis.

Tab. 7 reports the comparison in terms of load factor between the proposed macro-block model and the micro-block model of Orduña [20], highlighting the percentage difference; in Tab. 8, instead, there are the load factors provided by the other macro-block models and the percentage difference with respect to the micro-block model of Orduña [20]. In both cases, the comparison is possible only with reference to a single-storey wall (Sets 11-14), accounted by all the models considered. Multi-storey walls are, in fact, accounted only by the model developed by Speranza [28] and the comparison with the proposed model is reported in Tab. 9 with reference to a five-storey wall, having constant inter-storey height. Looking at the results related to Sets 11 and 12 in Tabs. 7 and 8, it is possible to derive that when $t \leq 1$, i.e. for stocky walls, the load factor is mostly not influenced by this parameter. Besides, the load factors obtained from the proposed model (Tab. 7) and the Speranza's model [28] (Tab. 8) are very close to that of the micro-block model and by the safe side too. Instead, the load factors of the other macro-block models (Tab. 8) provide higher percentage differences with respect to the micro-block model, up to $23 \%$ for the model of De Buhan and de Felice [29].

\begin{tabular}{cccc}
\hline Set & Micro-block model [20] & Proposed macro-block model & $\% \lambda$ Var. \\
11 & 0.69 & 0.65 & -5.8 \\
12 & 0.68 & 0.65 & -4.4 \\
13 & 0.49 & 0.49 & \pm 0.0 \\
14 & 0.26 & 0.26 & \pm 0.0 \\
\hline
\end{tabular}

Table 7: Micro-block model [20] vs. the proposed macro-block model. Load factors and percentage differences.

\begin{tabular}{ccccccc}
\hline Set & Orduña $[20]$ & $\% \lambda$ Var. & De Buhan and de Felice $[29]$ & $\% \lambda$ Var. & Speranza $[28]$ & $\% \lambda$ Var. \\
11 & 0.75 & +8.7 & 0.53 & -23.2 & 0.67 & -2.8 \\
12 & 0.65 & -4.4 & 0.53 & -22.1 & 0.67 & -1.5 \\
13 & 0.50 & +2.0 & 0.43 & -12.2 & 0.52 & +6.1 \\
14 & 0.25 & -3.8 & 0.31 & +19.2 & 0.27 & +3.8 \\
\hline
\end{tabular}

Table 8: Load factors provided by literature macro-block models and percentage difference with the micro-block model.

\begin{tabular}{cccc}
\hline Set & Proposed model & Speranza $[28]$ & $\% \lambda$ Var. \\
15 & 0.47 & 0.52 & 10.64 \\
16 & 0.47 & 0.48 & +2.13 \\
17 & 0.47 & 0.37 & -21.28 \\
\hline
\end{tabular}

Table 9: Load factors provided by the proposed model and that of Speranza [28] related to Sets 15, 16 and 17. 
Moreover, for the proposed macro-block model, a doubled value of the unit shape ratio $m$, accounted by Sets 13 and 14 (Tab. 7), involves a reduction of the load factor to about half. The values obtained, on the other hand, exactly coincide with those obtained from the micro-block model. The macro-block models of Orduña and Speranza confirm the same trend with little percentage differences with respect to the micro-block model. The model of De Buhan and de Felice, instead, is less sensible to the parameter $m$ and its percentage variation with respect to the micro-block model is more evident. All the models, however, highlight the same trend, i.e. the load factor decreases as the unit shape ratio $m$ increases. This trend can be explained with the reduction of the frictional resistances due to reduction of the overlapping length of the unit blocks.

Thus, summarizing the mentioned results related to a single-storey wall, it arises that the proposed model together with the model of Speranza [28] are more reliable with respect to the other accounted macro-block models, since they provide limited percentage differences with respect to the micro-block model of Orduña [20], assumed as a reference. These results are also better displayed in Fig. 5.

$\lambda$
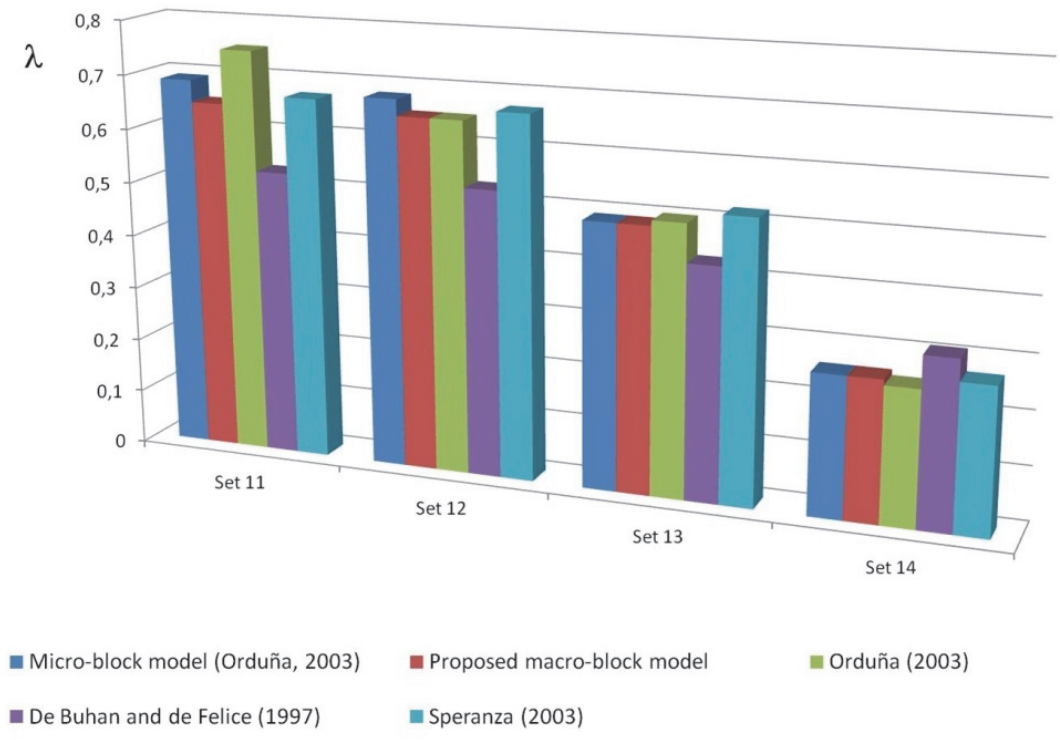

Figure 5: Load factors provided by the literature vs. the proposed macro-block model.

As previously anticipated, with reference to multi-storey walls the proposed model is comparable only with that developed by Speranza [28], since the other macro-block models do not account for this case. Thus, the results related to Sets 15, 16, 17 are reported in Tab. 9. It emerges that while the load factor of the proposed model is not influenced by the parameter $p$ accounting for the overloading, that provided by Speranza [28] decreases with increasing $p$. This trend of the latter model is mainly due to the absence of the contribution $F_{q}$ of the frictional resistance which therefore cannot counteract the overturning moment of the increasing overloading, as is possible with the proposed model.

\section{CONCLUSIONS}

I $\mathrm{n}$ this work, the mixed rocking-sliding mechanism of multi-storey walls due to in-plane horizontal actions has been analyzed; to this aim, a macro-block model has been adopted, schematizing the geometry of the mechanism through a single crack line where the frictional resistances and the relative displacements between the macro-blocks are concentrated. Hence, the kinematic approach of limit analysis has been used to evaluate the inclination of the crack line and the position of the rotational hinge along the height of the wall, assuming that it is always located in correspondence of the floor levels. With such a purpose, a routine of minimization of the load factor related to each possible position has been implemented, comparing the results in order to choose the minimum value. The innovative aspect of the study is mainly related to the modelling of the resultant frictional resistance involved in a mixed mechanism with rocking and sliding; in fact, a criterion, based on the inclination angle of the crack line, is adopted, under the assumption of Coulomb 
dry frictional contact with non-associated flow rule. This criterion, experimentally and numerically validated in previous works is now generalized to the analysis of a multi-storey masonry wall.

A numerical analysis has been performed in order to test the sensitivity of the proposed model to the main geometrical parameters, as the shape ratios related to the wall and unit block and the number of rows; the overloading condition and the friction coefficient have been also taken into account. The analysis has highlighted that the overloading ratio and the number of rows are substantially irrelevant both on the load factor and the inclination angle $\alpha_{c}$ of the crack line; the unit shape ratio $m$, instead, influences both these parameters that result decreasing with increasing values of $m$. Moreover, the wall shape ratio $t$, has a key role in the rocking-sliding mechanism of a slender multi-storey wall $(t>1)$ while it is quite irrelevant for squat walls $(t<1)$. In the first case, in fact, increasing values of $t$ imply increasing slenderness of the wall and decreasing load factors $\lambda$. This parameter, instead, results increasing with increasing values of the friction coefficient, as expected.

Lastly, the proposed model has been validated through the comparison against a micro-block and other macro-block models existing in the literature. The comparison has been performed in terms of both the load factor and the failure mode and with reference to key parameters as wall shape ratio, unit shape ratio, number of rows and overloading condition. It emerged that the proposed model provides results very close to the micro-block model, assumed as a reference, and can approach the "exact" solutions much better than the other compared macro-block models. These results can be very promising because can be obtained with lower computational effort than the micro-block modelling approaches, suggesting the possibility of using the proposed model also for extensive evaluations of the vulnerability of the ancient urban centres.

\section{ACKNOWLEDGMENTS}

$\mathrm{T}$ he authors acknowledge the sponsorship of the Italian Civil Protection, through the RELUIS Project-Line: Masonry Structures (2018).

\section{REFERENCES}

[1] Page, A.W. (1981). The biaxial compressive strength of brick masonry, P. I. Civil Eng. Pt. 1, 71 (2), pp. 893-906.

[2] Mann, W. and Muller, H. (1982). Failure of shear-stressed masonry. An enlarged theory, tests and application to shear walls, P. Brit. Ceramic Soc., 30, pp. 223-235.

[3] Dialer, C. (1991). Some remarks on the strength and deformation behaviour of shear stressed masonry panels under static monotonic loading, Proc. 9th International Brick/Block Masonry Conference, Berlin (Germany), pp. 276-283.

[4] Ceradini, V. (1992). Models and experimental tests for the study of historical masonry, Ph.D. Thesis., University of Roma La Sapienza, Roma, Italy.

[5] Roca, P., Cervera, M., Gariup, G. and Pelà, L. (2010). Structural Analysis of Masonry Historical Constructions. Classical and Advanced Approaches, Arch. Comput. Methods Eng., 17, pp. 299-325. DOI: $10.1007 / \mathrm{s} 11831-010-9046-1$.

[6] Bui, T.T., Limam, A., Sarhosis, V. and Hjiaj, M. (2017). Discrete element modelling of the in plane and out of plane behaviour of dry joint masonry wall constructions, Eng. Struct., 136, pp. 277-94.

DOI: 10.1016/j.engstruct.2017.01.020.

[7] Cannizzaro, F., Pantò, B., Caddemi, S. and Caliò, I. (2018). A Discrete Macro-Element Method (DMEM) for the nonlinear structural assessment of masonry arches, Eng. Struct., 168, pp. 243-256. DOI: $10.1016 /$ j.engstruct.2018.04.006.

[8] Gilbert, M., Casapulla, C. and Ahmed, H.M. (2006). Limit analysis of masonry block structures with non-associative frictional joints using linear programming, Comput. Struct., 84(13-14), pp. 873-87.

DOI: $10.1016 /$ j.compstruc.2006.02.005.

[9] Mousavian, E. and Mehdizadeh Saradj, F. (2018). Automated detailing and stability analysis of under-construction masonry vaults, J. Archit. Eng. ASCE 24(3), art. no. 04018014. DOI: 10.1061/(ASCE)AE.1943-5568.0000314.

[10] Silva, L.C., Lourenço, P.B. and Milani, G. (2017). Nonlinear discrete homogenized model for out-of-plane loaded masonry walls, J. Struct. Eng.-ASCE, 143(9), art. no. 04017099. DOI: 10.1061/(ASCE)ST.1943-541X.0001831. 
[11] Kooharian, A. (1952). Limit analysis of voussoir (segmental) and concrete arches, J. American Concrete Institute, 24(4), pp. 317-28.

[12] Heyman, J. (1966). The stone skeleton, Int. J. Solids Struct., 2, pp. 249-79.

[13] Drucker, D.C. (1954). Coulomb friction, plasticity and limit loads, J. App. Mech., 21(1), pp. 71-4.

[14] Livesley, R.K. (1978). Limit analysis of structures formed from rigid blocks, Int. J. Num. Method Eng., 12, pp. 185371. DOI: $10.1002 /$ nme.1620121207.

[15] Casapulla, C. and Maione, A. (2016). Formulating the torsion strength of dry-stacked stone blocks by comparing convex and concave contact formulations and experimental results, Indian Journal of Science and Technology, 9(46), 1-7.c DOI: $10.17485 /$ ijst/2016/v9i46/107346.

[16] Casapulla, C. and Maione, A. (2018). Modelling the dry-contact interface of rigid blocks under torsion and combined loading: concavity vs. convexity formulation, Int. J. Nonlin. Mech., 99, pp. 86-96. DOI: 10.1016/j.ijnonlinmec.2017.11.002.

[17] Sarhosis, V., Garrity, S.W. and Sheng, Y. (2015). Influence of brick-mortar interface on the mechanical behaviour of low bond strength masonry brickwork lintels, Eng. Struct., 88, pp.1-11. DOI: 10.1016/j.engstruct.2014.12.014.

[18] Sassu, M., Giresini, L., Bonannini, E. and Puppio M.L. (2016). On the Use of Vibro-Compressed Units with BioNatural Aggregate, Buildings, 6(3), art. no. 40. DOI: 10.3390/buildings6030040.

[19] D'Ayala, D. and Speranza, E., 2003. Definition of collapse mechanisms and seismic vulnerability of masonry structures, Earthq. Spectra, 19(3), 479-509. DOI: 10.1193/1.1599896.

[20] Orduña, A. (2003). Seismic assessment of ancient masonry structures by rigid blocks limit analysis, Ph.D. Thesis, University of Minho, Guimarães, Portugal.

[21] Casapulla, C., Maione, A., Argiento, L.U. and Speranza E. (2018). Corner failure in masonry buildings: an updated macro-modeling approach with frictional resistances, Eur. J. Mech. A-Solid, 70, pp. 213-225. DOI: 10.1016/j.euromechsol.2018.03.003.

[22] Casapulla, C. and Argiento, L.U. (2018). In-plane frictional resistances in dry block masonry walls and rocking-sliding failure modes revisited and experimentally validated, Compos. Part B-Eng., 132, pp. 197-213. DOI: 10.1016/j.compositesb.2017.09.013.

[23] Lagomarsino, S. and Podestà, S. (2004). Seismic vulnerability of ancient churches: I. Damage assessment and emergency planning, Earthq. Spectra, 20(2), pp. 377-94. DOI: 10.1193/1.1737735.

[24] Pantò, B., Giresini, L., Sassu, M. and Caliò, I. (2017). Non-linear modeling of masonry churches through a discrete macro-element approach, Earthq. Struct., 12(2), pp. 223-236. DOI: 10.12989/eas.2017.12.2.223.

[25] Giresini, L., Sassu, M., Butenweg, C., Alecci, V. and De Stefano, M.D. (2017). Vault macro-element with equivalent trusses in global seismic analyses, Earthq. Struct., 12(4), pp. 409-423. DOI: 10.12989/eas.2017.12.4.409.

[26] Lagomarsino, S. (2015). Seismic assessment of rocking masonry structures, B. Earthq. Eng., 13(1), pp. 97-128. DOI: $10.1007 / \mathrm{s} 10518-014-9609-\mathrm{x}$.

[27] Casapulla, C. and Maione, A. (2017). Critical response of free-standing rocking blocks to the intense phase of an earthquake, International Review of Civil Engineering, 8(1), pp. 1-10. DOI: 10.15866/irece.v8i1.11024.

[28] Speranza, E. (2003). An integrated method for the assessment of the seismic vulnerability of historic buildings, Ph.D. Thesis, University of Bath, UK.

[29] De Buhan, P. and De Felice, G. (1997). A homogenisation approach to the ultimate strength of brick masonry, J. Mech. Phys. Solids, 45(7), pp. 1085-104. DOI: 10.1016/S0022-5096(97)00002-1. 